\title{
Research on Gold in Catalysis
}

\author{
CURRENT TRENDS DISCUSSED AT A RECENT SYMPOSIUM
}

\author{
Israel E. Wachs \\ Corporate Research-Science Laboratories, Exxon Research and Engineering Co., Linden, N.J., U.S.A.

\begin{abstract}
The first symposium on the catalytic aspects of gold was held at the Eighth North A merican Meeting of the Catalysis Society, May 3-4, 1983 at the Franklin Plaza Hotel, Philadelphia. The symposium, co-sponsored by the Gold Institute, brought together widely scattered research efforts on catalysis by gold. Thissymposium advanced our understanding of the role and characteristics of gold catalysts by focusing attention on the research carried out in this somewbat neglected field.
\end{abstract}

J. Schwank of the University of Michigan, Ann Arbor, Michigan, reviewed the use of gold in heterogeneous catalysis. Only a brief description of this presentation will be given here since a more detailed description of this review is given in the adjoining article by Professor Schwank. The adsorption and reactivity of small molecules on gold surfaces was discussed in great detail. Carbon monoxide, ethylene, and acetylene are reversibly adsorbed at room temperature on gold. Hydrogen is not adsorbed on gold at room temperature, and elevated temperatures $\left(677-977^{\circ} \mathrm{C}\right)$ are required to dissociate hydrogen into atomic hydrogen. Oxygen is reversibly and irreversibly adsorbed on gold. Gold catalyzes the $\mathrm{H}_{2}-\mathrm{D}_{2}$ exchange reaction and other reactions requiring hydrogen (olefin hydrogenation, reduction of nitric oxide, skeletal isomerization, and alkyl transfer), even though hydrogen does not readily chemisorb on gold. Gold is aselective oxidation catalyst, especially in the oxidation of alcohols to aldehydes. The catalytic properties of gold are modified by the support used (support effect). For many catalystsystemsalloying with gold has a beneficial effect upon the selectivity of the resultant catalyst, but is usually accompanied by a decrease in activity. The structural arrangement of certain supported bimetallic gold clusters isstrongly influenced by the support. The active sites ingold catalysis are still not well understood and the role played by surface defects, electron deficient gold sites, and other surface phenomena are not yet clear. J. Schwank's excellent review provides a long needed perspective on catalysis by gold.

\section{Cluster Compounds}

D.M.P. Mingos of the University of Oxford, Oxford; United Kingdom, reported on the chemistry of gold cluster compounds. A wide range of these compounds containing 2 to 13 gold atoms can besynthesized. The solid structures of the goldcluster compounds have been determined by singlecrystal $\mathrm{X}$-ray diffraction and solution structures by ultraviolet-visible reflectance spectroscopy. The number of gold atoms in a gold cluster compound is controlled via cluster addition orcluster degradation reactions. The progress of such reactions in solution has been monitored in situ with ultraviolet. visible reflectance spectroscopy. The gold cluster geometry and electronic configuration depend on the specific ligands present in the compound, and the oxidation state of the gold atoms in the various gold cluster compounds is intermediate between 0 and +1 . Phosphorus-31 nuclear magnetic resonance of the gold cluster compounds in solution yields only one sharp NMR peak. This indicates that the gold atoms in the compounds are not rigid, but move around in solution, that is they arestereochemically non-rigid.

$\mathrm{X}$-ray photoelectron spectroscopy examination of the valence band of the gold cluster compounds demonstrated that the larger clusters have somemetallic character. This aspect of gold clusters will be reviewed by Dr. Mingos in the next issue of Gold Bulletin. Heteronuclear mixed metal cluster compounds of gold can also be synthesized (for example Os-Au, Fe-Au among others) via similar methods. The above data suggest that these gold clustercompounds may be ideal model compounds for the study of metal surfaces in heterogeneous catalysis.

\section{Supported Gold Cluster Catalysts}

G. Meitzner, G. Zhang, and M. Boudart of Stanford University, Stanford, California, presented their findings on the preparation and characterization of supported gold clusters. The supported gold catalysts were characterized by X-ray diffraction and by X-ray absorption spectroscopy (gold $\mathrm{L}_{\mathrm{III}}$ near edge structure and Extended $\mathrm{X}$-ray Absorption Fine Structure(EXAFS)). Gold was supported on Y-zeolite $\left(600 \mathrm{~m}^{2} / \mathrm{g}\right)$, prepared by ion-exchange, and magnesia $\left(325 \mathrm{~m}^{2} / \mathrm{g}\right)$, prepared by impregnation. The catalysts subsequently received a mild oxygen treatment and reduction in hydrogen. The magnesia support was found to stabilize the gold $(+3)$ towards reduction. The higher temperatures $\left(>200^{\circ} \mathrm{C}\right)$ needed to effect reduction of the gold supported on magnesia results in the formation of large gold clusters due to the mobility of gold atoms at these temperatures. In contrast, on the Y-zeolite support reduction at $\approx 25^{\circ} \mathrm{C}$ readily converts gold $(+3)$ to metallic gold, the cluster being smaller than $1.5 \mathrm{~nm}$ in size. The size of the supported gold clusters is dependent on the temperature of reduction as revealed by XRD andEXAFS characterization of the catalyst. The small gold particles are maintained on the Y-zeolite support up to $\approx 145^{\circ} \mathrm{C}$, and at higher reduction temperatures the gold comes out of the zeolite pores and agglomerates. The ability of the Y-zeolite supported gold to catalyze the oxidation of molecular hydrogen to water was investigated as a function of reduction temperature (25 to $265^{\circ} \mathrm{C}$ ). The reduced, small, gold clusters inside the zeolite pores are not catalytically active for the $\mathrm{H}_{2}-\mathrm{O}_{2}$ reaction. The gold clusters become catalytically active when they agglomerate, but too much agglomeration leads to a decay in activity (due to loss in effective 
surface area). The maximum activity was obtained at a reduction temperature of $200^{\circ} \mathrm{C}$. Although oxygen and hydrogen do not adsorb well on the gold surface the $\mathrm{H}_{2}-\mathrm{O}_{2}$ reaction proceeds readily over gold catalysts. This suggests that there is a synergistic interaction between oxygen and hydrogen that leads to the formation of water (that is, adsorbed molecular oxygen activates the adsorption of molecular hydrogen).

J. Lee and J. Schwank of the University of Michigan, Ann Arbor, Michigan, examined the adsorption of nitric oxide and carbon monoxide on supported gold catalysts with infrared spectroscopy. The gold catalysts were prepared by either impregnation of the supports (silica and magnesia) with chloroauric acid or potassium gold (I) cyanide, or by cationic exchange with $\mathrm{Au}(\mathrm{en})_{2} \mathrm{Cl}_{3}$. The infrared experiments were conducted in a glass vacuum cell connected to a gas handling system. The samples were pretreated in situ in flowing helium, reduced in hydrogen at $350^{\circ} \mathrm{C}$, and evacuated prior to introduction of the adsorption gases. The adsorption of carbon monoxide on the gold catalysts produces an infrared band in the region of 2115 to $2040 \mathrm{~cm}^{-1}$. The adsorption of nitric oxideon the gold catalysts does not give rise to an infrared band. The absence of this implies that the adsorbate is either dissociated, in a form that gives rise to a weak infrared signal, or in a state that is infrared-inactive. The presence of adsorbed nitric oxide on the gold catalysts was confirmed by the inability to adsorb carbon monoxide on catalysts pre-exposed to nitric oxide, and the inability of nitric oxide to displace preadsorbed carbon monoxide in to the gas phase. These observationsareconsistent with earlier kinetic studies which found that nitric oxide poisons the gold surface for the reaction:

$$
\mathrm{NO}+\mathrm{CO} \longrightarrow \mathrm{N}_{2} \mathrm{O}+\mathrm{CO}_{2}
$$

The reduction of nitric oxide over the gold/magnesia catalyst yields predominantly nitrogen, but the reduction of nitric oxide over the gold/silica catalyst yields predominantly ammonia. These different reaction selectivities were attributed to the different adsorbed states on these supported gold catalysts of nitric oxide which is dissociatively adsorbed on gold/magnesia and molecularly adsorbed on gold/silica catalysts. This study demonstrates the significant influence by the support upon the cataly tic properties of gold.

\section{Surface Chemistry}

P.B. Dorain, K.V. von Raben, and R.K. Chang of Yale University, New Haven, Connecticut, and B.L. Laube of United Technologies Research Center, East Hartford, Connecticut, have investigated the surface-enhanced Raman scattering of molecules adsorbed on gold and silver surfaces. The large enhancement of the Raman scattering efficiency of molecules adsorbed on gold and silver surfaces of the order of $10^{4}$ and $10^{7}$ respectively enables in situ examination of the vibrational modes of adsorbed molecules. The nature of the surface intermediates on the gold and silver surfacescan be determined from these vibrational modes. Adsorption of sulphur dioxide, nitric oxide and nitrogen dioxide on a preoxidized silver powder results in surface-enhanced Ramanscattering of adsorbed $\mathrm{SO}_{3}{ }^{2-}, \mathrm{NO}_{2}{ }^{-}$, and $\mathrm{NO}_{2}{ }^{-} / \mathrm{NO}_{3}{ }^{-}$, respectively. On preoxidized gold powders surfaceenhanced Raman scattering of adsorbed nitrogen and sulfur oxides was not detected. The reason for the absence of these Raman signals from the gold powders is not clear, but may be due to the presence of impurities on the gold surface which inhibit adsorption of gaseous molecules. The influence of surface impurities in gold is discussed later in this report.

The surface-enhanced Raman scattering of adsorbates on gold colloid surfaces, however, was detectable, demonstrating the existence of the enhancement factor for gold. The vibrational frequency of $2138 \mathrm{~cm}^{-1}$ in the presence of cyanide anions implies that the intermediate present on the gold colloid surface is $(\mathrm{CN})_{2}{ }^{2-}$. Addition of small quantities of nitric acid to the colloidal solution shifted the vibrational mode to $2180 \mathrm{~cm}^{-1}$ which implies that the adsorbed species changes to $\mathrm{CN}^{-}$in the presence of nitric acid. Surface-enhanced Raman scattering is alsocapable of providing in situ information about the gold electrode-electrolyte interface. The temporal evolution of the surface-enhanced Raman spectra of adsorbed $\mathrm{CN}^{-}$on the gold surface as the gold electrode is undergoing an electrochemical oxidation-reduction cycle was also presented. Additional surface-enhanced Raman scattering studies of gold surfaces should provide much needed insight in to the surface chemistry of gold.

D.R. Miller and M. Cates of the University of California-San Diego, La Jolla, California, discussed the surface information obtainable from the analysis of helium atoms scattered from surfaces. Low energy helium scattering of the (111) oriented gold surface was used to probe the relation between surface structure and surface phonon dispersion. A $22 \mathrm{meV}$ helium atom beam, with a resolution of 3 percent, wasscattered from the gold surface and the inelastic and elastic scattering events were monitored by a standard time-of-flight technique (time of arrival at a massspectrometer). The elastic scattering coherent diffraction data suggest that the (111) oriented gold surface is very smooth, but that a long range $(\approx 3.0 \mathrm{~nm})$ order exists which may be related to a reconstruction or buckling of the surface. The inelasticscattering data yield a phonon dispersion in agreement with lattice calculations at low frequency but deviating at the zone edge.

Information about the gas-surface interaction potential is also obtainable from such scattering experiments. The helium atoms scattered from the (111) oriented gold surface are primarily elastic, about 80 percent, and reveal a very smooth interaction potential for the helium-gold (111) system. The scattering of hydrogen molecules from the gold surface is very similar to that of helium atoms. For the scattering of deuterium molecules from the same gold surface, however, the elastic component is decreased and the inelastic component dominates. The deuterium-gold (111) interaction potential is very different to the smooth interaction potentials found for hydrogen molecules and helium atoms with the same (111) oriented gold surface. Furtherstudies withother gaseousmolecules 
should lead to a better understanding of the significant molecular parameters affecting the nature of the gas-surface interaction potential for the gold (111) surface.

R.J. Madix and D.A. Outka of Stanford University, Stanford, California, reported on the surface chemistry of a (110) oriented single crystal gold surface. Augerelectronspectroscopy (AES)analysis of the gold surface revealed that an impurity-free gold surface was difficult to obtain. The adsorption of oxygen on the gold surface was found to induce segregation of silicon from the bulk to the surface, and to oxidize the silicon to silica. The silica on the gold surface was removable only by argon ion sputtering. However, further exposure of oxygen to the gold surface was again found to segregateadditional bulk silicon to the gold surface. After weeks of many such cleaning cycles, the surface concentration of silica eventually diminished, and it was possible to study the surface chemistry of a clean gold surface.

These cleaning cycles, however, converted the (110) orientation of the gold surface to a predominantly (111) orientation. The adsorption of oxygen, formaldehyde, formic acid, acetylene and methanol, on the clean gold surface was studied in the temperature range $-125^{\circ} \mathrm{C}$ to $25^{\circ} \mathrm{C}$. All but formic acid failed to adsorb on the clean gold surface in this temperature range. However, this latter compound was reversibly adsorbed on the clean gold surface, and no decomposition to formate $\left(\mathrm{HCOO}_{\text {ads }}\right)$ was detected. The weakly adsorbed formic acid desorbed at about $-50^{\circ} \mathrm{C}$ with an activation energy of about $12 \mathrm{kcal} / \mathrm{mole}$. The temperature-programmed desorption spectra of formic acid as a function of coveragesuggested the formation of 2-dimensional islands of the adsorbate on the gold surface. The islands of formic acid form because of its weak interaction with the gold surface and because of the much stronger interactions between the adsorbed formic acid molecules. In future studies the authors intend to pre-adsorb oxygen atoms on the gold surface via pre-dissociation of the gas phase oxygen with a hot filament, and to study the oxidation reactions of formic acid, formaldehyde, acetylene and methanol via the adsorbed oxygen atoms on the gold surface. These surface reactions should follow the same reaction pathways previously reported for silver and copper surfaces, but with different kinetics.

\section{Gold Alloys}

C. Serrano and J.J. Carberry of the University of Notre Dame, Notre Dame, Indiana, presented their study on the oxidation of carbon monoxide over $\alpha$-alumina supported gold-silver alloys. The kinetics of the oxidation of carbon monoxide oversupported silver, and gold-silver alloys were measured in a well mixed gradientless reactor (Notre Dame Spinning Basket Reactor) at atmospheric pressure and at temperatures between 143 and $250^{\circ} \mathrm{C}$. The loading of silver on the low surface area $\alpha$-alumina support was maintained at 0.78 weight per cent throughout the study, and resulted in very large silver crystallites (a dispersion of 0.04 per cent). The alumina supported catalysts were reduced at $300^{\circ} \mathrm{C}$ in hydrogen for three hours prior to the oxidation of carbon monoxide. The kinetics of the oxidation of carbon monoxide over supported silver were found to be first order in carbon monoxide and inhibited by product carbon dioxide as shown:

$$
\mathrm{R}=\frac{\mathrm{kCO}}{\left(1+\mathrm{kCO}_{2}\right)}
$$

This rate expression suggests that carbon monoxide chemisorption is rate controlling on a surface partially covered by product carbon dioxide (probably present as a surface carbonate). The above kinetic rate expression was unchanged when using up to 9 a tomic per cent gold in the silver. The inhibition of the oxidation kinetics by carbon dioxide becamestrongerwithalloyspossessing greater than 9 atomic percent gold in silver, and the order in inhibition by carbon dioxide increased to negative second order for 38 atomic per cent gold in silver:

$$
\mathrm{R}=\frac{\mathrm{k}^{\mathrm{C}} \mathrm{CO}}{\left(1+\mathrm{k}^{\mathrm{1}} \mathrm{CO}_{2}\right)^{2}}
$$

This rate expression suggests the surface reaction between $\mathrm{O}_{\text {ads }}$ and $\mathrm{CO}_{\mathrm{ads}}$ is rate controlling on a surface partially covered by product carbon dioxide (Langmuir-Hinshelwood kinetics). The aluminasupported gold catalyst wasfound to be inactive for carbon monoxide oxidation in this temperature range, and only became active at $320^{\circ} \mathrm{C}$. The kinetics of carbon monoxide oxidation described above suggest that the role of gold in gold-silver alloys during the oxidation of carbon monoxide is that of a geometric diluent, causing the rate controlling reaction step to change from that of carbon monoxide chemisorption to that of surface reactions between adsorbed oxygen and carbon monoxide. Additional insight into the gold-silversystem was provided by X-ray photo-electron spectroscopy (XPS) studies of the surface composition of a series of unsupported gold-silver alloys. The XPS data revealed that a very large bulk concentration of gold is required before the surface concentration of gold becomes significant. The XPS data account for the large bulk concentrations of gold required to influence the carbon monoxide oxidation reaction over gold-silver alloys.

V.N. Mallikarjuna Rao of E.I, du Pont De Nemours and Company, Inc., Wilmington, Delaware, discussed the advantages of gold-silver alloy catalysts over conventional silver catalysts in the synthesis of formaldehyde via methanol oxidation. Unsupported silver catalysts are widely used for the commercial production of formaldehyde from methanol. The silver catalyzed reaction is conducted at temperatures of 550 to $750^{\circ} \mathrm{C}$ in an adiabatic reactor. Essentially complete conversion of methanol is achieved at these temperatures in a two stage reactor. The main by-product of the reaction is carbon dioxide, with carbon monoxide and methyl formate appearing as the minor by-products. Gold catalysts, however, are highly selective to formaldehyde, but not as active as silvercatalysts for the oxidation of methanol. The major by-product 
in the gold-catalyzed synthesis of formaldehyde is methyl formate, carbon dioxide and carbon monoxide being absent from the product stream. Furthermore, gold catalysts, unlike silver are notcapable of oxidizing formaldehyde to carbon oxides.

Higher formaldehyde yields, however, are obtained from the oxidation of methanol overgold-silveralloy catalysts than fromeither silver or gold catalysts. The optimum yield of formaldehyde is obtained for gold/silver alloycatalystscontaining 40 to 60 atomic per cent gold. Higher concentrations of gold in the alloy catalyst yield unacceptable amounts of carbon monoxide and methyl formate during the oxidation of methanol. At low methanol conversions (about 50 per cent) a formaldehyde selectivity improvement of 1 to 2 percent isobtained with the gold-silvercatalysts over that obtained using a silver catalyst. A formaldehyde selectivity improvement of 3 to 5 per cent is obtained with the alloy catalyst at a methanol conversion level of greater than 90 per cent, using a two-stage process.

The formaldehyde selectivity improvement obtained with goldsilver catalysts during methanol oxidation arises from two simultaneous effects due to the addition of gold to the silver catalyst:

(1) The addition of gold decreases the rate of the undesirable oxidation of formaldehyde to carbon oxides relative to the rate of methanol oxidation to formaldehyde. This is especially important at highconversions of methanol where the concentration of formaldehyde in the gas-phase is high and the concentration of methanol is low.

(2) The lower activity of gold catalysts for this oxidation reaction spreads out the very narrow reaction zone in the adiabatic reactor and lowers the temperature of operation. The lower reaction temperature minimizes the combustion of formaldehyde due to diffusion (mass transfer limitations).

Several process improvementsinaddition to the formaldehyde selectivity improvement are also realized with the use of gold-silver alloy catalysts. The operating lifetimes of the alloy catalysts are significantly longer than those of commercially used silvercatalysts. This is due partly to a reduced rate of carbon deposition on the catalytic surface, and partly to the diminished tendency towards sintering. The selectivity for formaldehyde production during methanol oxidation decreases with time for all catalysts but the rate of decrease for the gold-silver catalysts is much slower than the corresponding rate of decrease for silvercatalysts. The loweroperating temperature of the gold-silver catalyst requires less energy to maintain the reactor temperature. Overall, the performance of goldsilver catalysts for the oxidation of methanol to formaldehyde is superior to that of conventional silver catalysts.

M.A. Adams, L.C. Roselaar, and D.E. Webster of the Polytechnic of the South Bank, London, United Kingdom, presented a study of the oxidation of methanol overgold, palladium and gold-palladium alloy catalysts. The catalysts consisted of electrically heated wires the temperatures of which were determined by measuring the electrical resistance. The product stream was analyzed for formaldehyde (determined calorimetrically), carbon monoxide and carbon dioxide (determined by infrared spectroscopy) and hydrogen and water (determined by gas chromatography). The oxidation of methanol over the gold catalyst occurred at temperatures above $350^{\circ} \mathrm{C}$ and yielded formaldehyde, water, and small amounts of hydrogen (about 10 per cent); carbon monoxide and carbon dioxide were not detected. The methanol oxidation kinetics over the gold catalyst were found to be zero order in oxygen, independent of oxygen partial pressure, and first order in methanol. At high methanol concentrations, the kinetics of formaldehyde formation also became independent of the methanol partial pressure. The activation energy of this reaction was found to be $32 \mathrm{kcal} / \mathrm{mole}$. This kinetic dependence suggested to the investigators that the oxidation of methanol over gold occurs berween adsorbed molecular oxygen and adsorbed methanol.

Palladium became active for the oxidation of methanol at temperatures above $150^{\circ} \mathrm{C}$ (approximately $200^{\circ} \mathrm{Clower}$ than gold). The methanol oxidation kinetics were found to be first order in oxygen and methanol partial pressures. The kinetics were ascribed to the surface reaction between adsorbed oxygen atomsand adsorbed methanol. The oxidation of methanol over the palladium catalyst yielded primarily carbon dioxide and water, small amounts of formaldehyde and hydrogen, and no carbon monoxide. The absence of this latter in the product stream was attributed to the strong chemisorption of carbonmonoxide on palladiumand itssubsequent surface oxidation to carbon dioxide. Carbon monoxide, however, became a significant reaction product during methanol oxidation over gold-palladium alloy catalysts. The addition of gold to palladium, in concentrations of up to about 40 atomic percent gold, increased the yield of carbon monoxide and increased the minimum temperature required to oxidize methanol. The enhanced formation of carbon monoxide over gold-palladium alloys was attributed to the weak interaction between carbon monoxide and gold, and the ability of carbon monoxide to desorb from the gold sites on the gold-palladium surface prior to further oxidation to carbon dioxide. This study demonstrates that the alloying of gold with other metalscan lead to reaction products not normally formed with the pure components.

\section{Electrocatalysis}

B. Beden, I. Cetino, A. Kahyaoglu, and C. Lamy of Universitéde Poitiers, Poitiers, France, discussed the electrochemistry of gold electrodes. In their experiments using cyclic voltammetry, each voltammogram is characteristic of the electrode material and the state of the electrode surface. The electrolysis of water, for example, exhibits a hydrogen adsorption stage and an oxygen evolutionstage over most metal electrodes, but for gold there is no hydrogen adsorption step, only an oxygen evolutionstage. The oxygen monolayer on the gold electrode surface inhibits the oxidation of formic acid until the oxygen layer is evolved from the gold surface.

The electrocatalytic oxidation of ten organic compounds containing two carbon atoms (ethane, ethanol, ethylene-glycol, 
acetaldehyde, glycolaldehyde, glyoxal, acetic acid, glycolic acid, glyoxylic acid and oxalic acid) was investigated on gold electrodes in acid and alkaline media using cyclic voltammetry. Gold behaves as a poor electrocatalyst in an acidic medium (perchloric acid) except in the oxidation of glyoxylic and oxalic acids. In alkaline medium (sodium hydroxide), however, gold is an excellent electrocatalystand even performs better than platinum, particularly for the aldehyde compounds. The relatively poor performance of the platinum electrode is thought to be related to the poisoning of the platinum surface by stable reaction intermediates. The degree of poisoning of the gold surface is substantially reduced relative to platinum because the reaction products desorb more easily from the gold surface. The differences between the gold electrode surface in alkaline and acidic media are presently not understood. In situ infrared studies in progress should provide additional insight into theorigin of this difference. This study clearly demonstrates that gold is an active and selective electrocatalyst for oxidation reactions in alkaline media.

K.V. Rao and C.B. Roy of the Indian Institute of Technology, Kharagpur, India, investigated the electrocatalytic oxidation of methanol, ethanol, isopropanol, glycol and glycerol on goldplatinum alloy electrodes. The oxidation of these oxygenates was examined with the galvanostatic steady-state method. Ethanol was oxidized insulphuric acid and the other compounds in an alkaline medium. All the oxygenated compounds oxidized more readily over the gold-platinum alloy electrodes, containing up to 75 atomic per cent gold, than over either platinum or gold electrodes, In all cases the electrocatalytic activity exhibited a maximum for the alloy containing 35 atomic per cent gold. The maximum activity of the gold-platinum electrode is approximately an order of magnitude higher than that of the platinum electrode on a basis of unit mass of catalyst. The enhanced electrocatalytic activity of the goldplatinum electrodes relative to the platinum electrode is still maintained when the data are corrected for differences in surface area of the alloy electrode compared to the pure platinum. Thissuggests that a synergistic interaction between platinum and gold is responsible for the enhanced electrocatalytic oxidation activity of oxygenated compounds over gold-platinum alloy electrodes. The enhanced catalytic activity of the alloy electrodes is consistent with the explanation earlier proposed by Beden et al:: the platinum electrode surface is poisoned by stable reaction intermediates and the reaction products desorb more easily from the gold surface. Thus, the high activity of the gold-platinum alloy surface may be due to the rapid adsorption of reactants at the platinum sites and the facile desorption of reaction products from the gold sites.

\section{Summary}

The papers presented in this symposium demonstrate that gold possesses scientifically interesting and industrially important catalytic properties. Clearly, gold has its greatest impact in the area of oxidation catalysis, as reflected in the number of papers in this symposium addressing the subject. The use of gold as an electrocatalyst appears to be an area with much potential. The surface chemistry, in particular that pertaining to adsorption, desorption, surface reaction, and surface intermediates of these gold-catalyzed reactions, however, is poorly understood at present. For example, do the oxidation reactions over gold catalysts proceed with molecularly adsorbed oxygen, dissociatively adsorbed oxygen, or both (depending on reaction conditions)? Hopefully, further research in this area will advance our understanding of the surface chemistry of gold catalysts.

Several of the papers in thissymposium showed that the selectivity of many reactions is affected by gold alloy catalysts and by support materials. Gold alloy catalysts modify the relative reactivity of the reactants and products leading, in somecases, to improved selectivity to the desired products. The somewhat lower activity of gold alloy catalysts, with the exception of the gold alloy electrocatalysts, can also have a beneficial effect on the selectivity of some reactions because of the decrease in mass transfer limitations. The observation that support materials influence the catalytic behaviour of gold is very significant, and much more research is needed to understand this phenomenon.

The somewhat lower activity of gold catalysts requires that special attention be paid to impurities, the presence of which in gold catalysts was discussed by several speakers and which probably accounts for some of the contradictions that exist in the gold catalysis literature. Many of the researchers requested that the Gold Institute provide, on a loan basis, a source of high purity gold to overcome this problem and help tostandardize research in the area of gold catalysis. Such an approach will have a positive effect on the advancement of our understanding of the catalysis of gold. The lack of financial assistance for research in gold catalysis was also cited as a major obstacle.

\section{Reader Opinion Survey - a Reminder}

A Reader Opinion Survey was sent to all recipients of Gold Bulletin as an insert in the last issue, July 1983. The purpose of this questionnaire is to elicit readers views on how relevant and valuable Gold Bulletin is to their particular activities. From these views it is intended to adjust and improve the journal in those areas where deficiencies have become apparent. Although a significant response and many constructive comments have already been received it is obvious that the higher the response rate the more representative of the whole readership will be the result of the survey. It would be much appreciated therefore if, in the event that you have not yet responded, you could look through your July 1983 issue and, if your questionnaire is still available, fill it in and return it to the address shown.

Thank you for your co-operation. 